\title{
A NOVEL METHOD OF CONSTRUCTING OPEN 3D VIRTUAL SIMULATION PLATFORM FOR ROBOT
}

\author{
Ning Tingzho ${ }^{1}$, Fu Ling ${ }^{1}$, Zhang Jingzhi ${ }^{1}$ \\ ${ }^{1}$ College of Mechanical and Electrical Engineering, Zaozhuang University, Zaozhuang 277160, China \\ Email: zjzsd2003@163.com
}

\begin{abstract}
For 3D motion simulation and real-time online control of manipulator, a novel which is fast and efficient method that constructing an open 3D virtual simulation platform for manipulator was presented. The method was carried by the MATLAB software, modeling software of SolidWorks, Robotics Toolbox, MATLAB GUI interaction design, 3D animation and the displayed technology of MATLAB, and it was applied to construct a good visualization, powerful function of the input and output, which completed a 3D virtual simulation platform for the manipulator. Taking the six DOF modular manipulator UR5 as an example, the implementation steps of this method were detailed described, while a high-level open 3D virtual simulation platform for the manipulator was achieved. Part of the simulation graphs were displayed, and the 3D virtual simulation platform for the manipulator was constructed quickly with good usability and high maneuverability by the method. As a result, it proved the effectiveness of our method to meet the needs of scientific research and teaching.
\end{abstract}

Keywords: Virtual Simulation Platform; Modular Manipulator; Visualization; 3D Animation;

\section{Introduction}

With the continuous development of automation technology, the application of robot technology in vehicle manufacturing, electronic device manufacturing, palletizing, spraying and welding and other operations is becoming more and more widespread. Due to the expensive and large homework, it is necessary to develop 3D virtual simulation platform for robots with the high degree of visualization in teaching and scientific research.

Many scholars have studied the robot simulation technology in domestic and abroad, including 3D simulation software for scientific research and teaching, and they are mainly concentrated in the overseas [1-5].

China is focusing on the study of [6-11], which is developed by the MATLAB software and OpenGL. The former is mostly a stick figure model to illustrate its kinematics principle and hard to express the ontology of collision and interference problems, the latter has good programmability and portability, but it is mostly for animation demonstration without real-time online control function.

There have 3D simulation systems for industrial applications [12-14], most of which focus on aerospace, automobile manufacturing and other aspects. However, these simulation systems are relatively closed, with poor universality and limited application scope.
Literature [15] has proposed a method of building opened robot simulation environment that developed based on MATLAB software platform. However, the way in establishing a mechanical arm will lead to the out-of-order scattered in the world coordinate system.

Therefore, the construction efficiency of robot simulation platform directly affects the follow-up work, and it becomes important for the rapid construction of robot 3D virtual simulation platform.

Aiming at the deficiency of the research and taking the literature [15] to construct the robot simulation platform, an open method for constructing 3D virtual simulation platform is put forward. Taking the 6-dof modular robot arm UR5 as an example, the 3D virtual simulation platform was constructed quickly in the paper.

\section{Construction Process of a Virtual Simulation Platform}

The method proposed in this paper is based on the MATLAB software environment. Solid Works for 3D modelling software and robot toolbox are used as the implementation tools. In addition, 3D display technology of MATLAB is also combined to realize the rapid construction of virtual platform.

The construction process of virtual platform can be divided into three steps, that is, the reconstruction of entity model, the import of model STL file and the model display and animation demonstration. 


\subsection{Model reconstruction of the robot arm}

In order to realize the rapid construction of robot simulation platform, an efficient and opened 3D virtual simulation construction method is proposed based on the literature [15].

Fig. 1 is the robot UR5 by the research group. For constructing an arm simulation model, in this paper, the mechanical arm model and DH method are shown in fig. 2 and the DH parameters of mechanical arm are shown in table 1 .

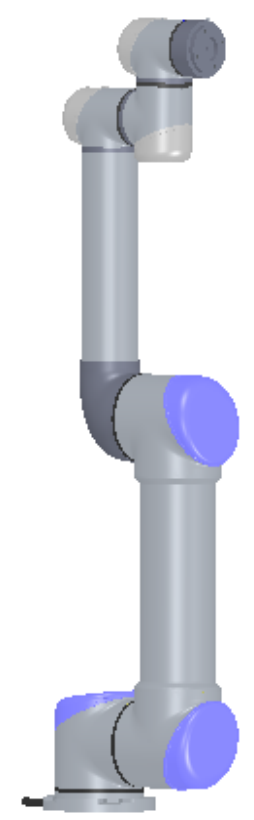

Figure 1: Model of the UR5 collaboration robot

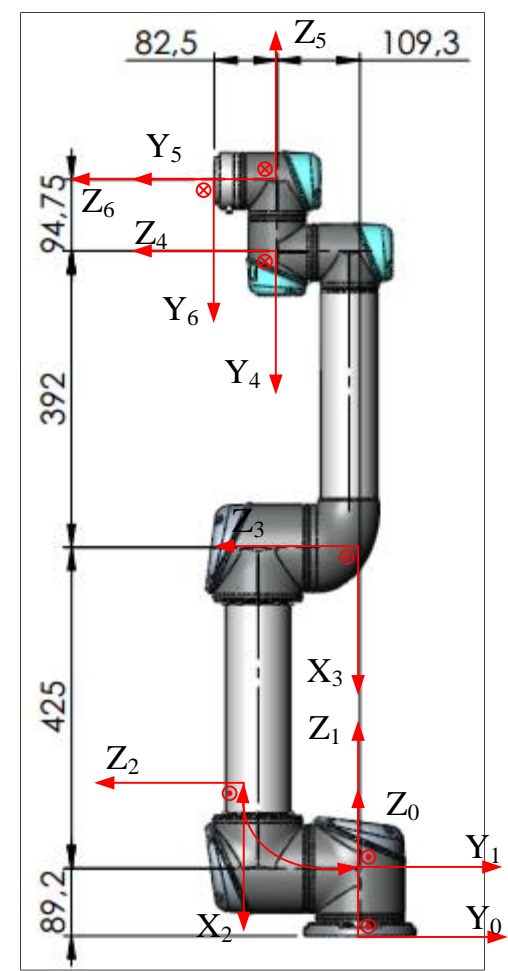

Figure 2: The coordinate frame of the manipulator
Table 1 The DH parameter of the manipulator

\begin{tabular}{|c|c|c|c|c|c|}
\hline Joint & $\begin{array}{c}\theta_{i} \\
/\left(^{\circ}\right)\end{array}$ & $\begin{array}{c}\alpha_{i-1} \\
/\left(^{\circ}\right)\end{array}$ & $\begin{array}{c}a_{i-1} \\
/(\mathrm{mm})\end{array}$ & $\begin{array}{c}d_{i} \\
/(\mathrm{mm})\end{array}$ & $\begin{array}{c}\text { Range } \\
/\left(^{\circ}\right)\end{array}$ \\
\hline 1 & $\theta_{1}$ & 90 & 0 & 89.2 & $-360 \sim+360$ \\
\hline 2 & $\theta_{2}$ & 0 & 425 & 0 & $-360 \sim+360$ \\
\hline 3 & $\theta_{3}$ & 0 & 392 & 109.3 & $-360 \sim+360$ \\
\hline 4 & $\theta_{4}$ & -90 & 0 & 0 & $-360 \sim+360$ \\
\hline 5 & $\theta_{5}$ & 90 & 0 & 94.75 & $-360 \sim+360$ \\
\hline 6 & $\theta_{6}$ & 0 & 0 & 82.5 & $-360 \sim+360$ \\
\hline
\end{tabular}

Then, the paper uses the modified DH method to establish each link coordinate system. For the base of part A, origin coordinate system $x_{0} y_{0} z_{0}$ is located in the center of part $\mathrm{A}$. In part $\mathrm{B}$, the coordinate system $x_{1} y_{1} z_{1}$ is located in the joint axis intersection point 1 and 2. In part C, coordinate system $x_{2} y_{2} z_{2}$ is located in joint 1 and 3 axis intersection point. For part $\mathrm{D}$, coordinate system $x_{3} y_{3} z_{3}$ is set in joint 4 and 5 axis intersection point. For part E, coordinate system $x_{4} y_{4} z_{4}$ is set in the joint axis intersection point 4 and 5 joints. For part $\mathrm{F}$, coordinate system $x_{5} y_{5} z_{5}$ is located in the joint 5 and 6 axis intersection point. For part $\mathrm{G}$, coordinate system $x_{6} y_{6} z_{6}$ in fig. 3 is set in the center of the part G.

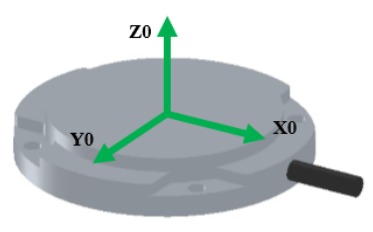

(A)

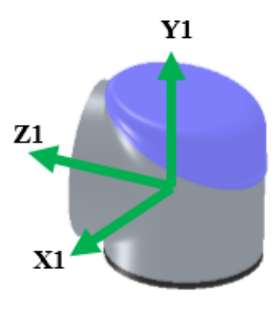

(B)

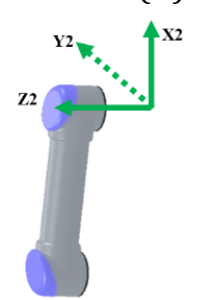

(C)

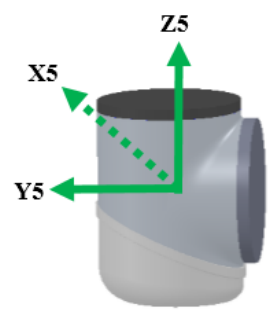

(F)

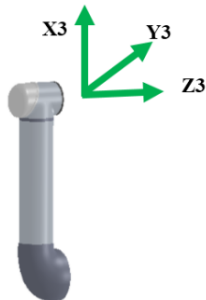

(D)

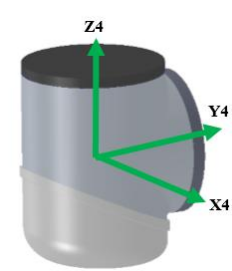

(E)

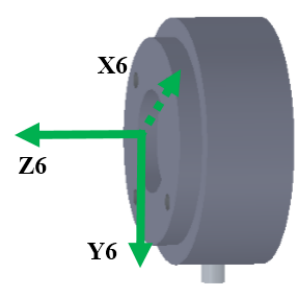

(G)

Figure 3: Coordinate reconstruction result of the split parts 


\subsection{Model import for robot arm}

After the coordinate system reconstruction of the manipulator, the model of the manipulator is imported into MATLAB to build its 3D virtual simulation platform. Since MATLAB software cannot directly recognize $\mathrm{CAD}$ graphic files, the files are transformed to the style ASCII of STL (stereo lithography).

Furthermore, the program function [fout, vout, cout]=stlread (filename) for importing STL model is written in MATLAB, and the related .mat file is achieved. The following is the working principle of specific flow chart, and it is shown in fig.4.

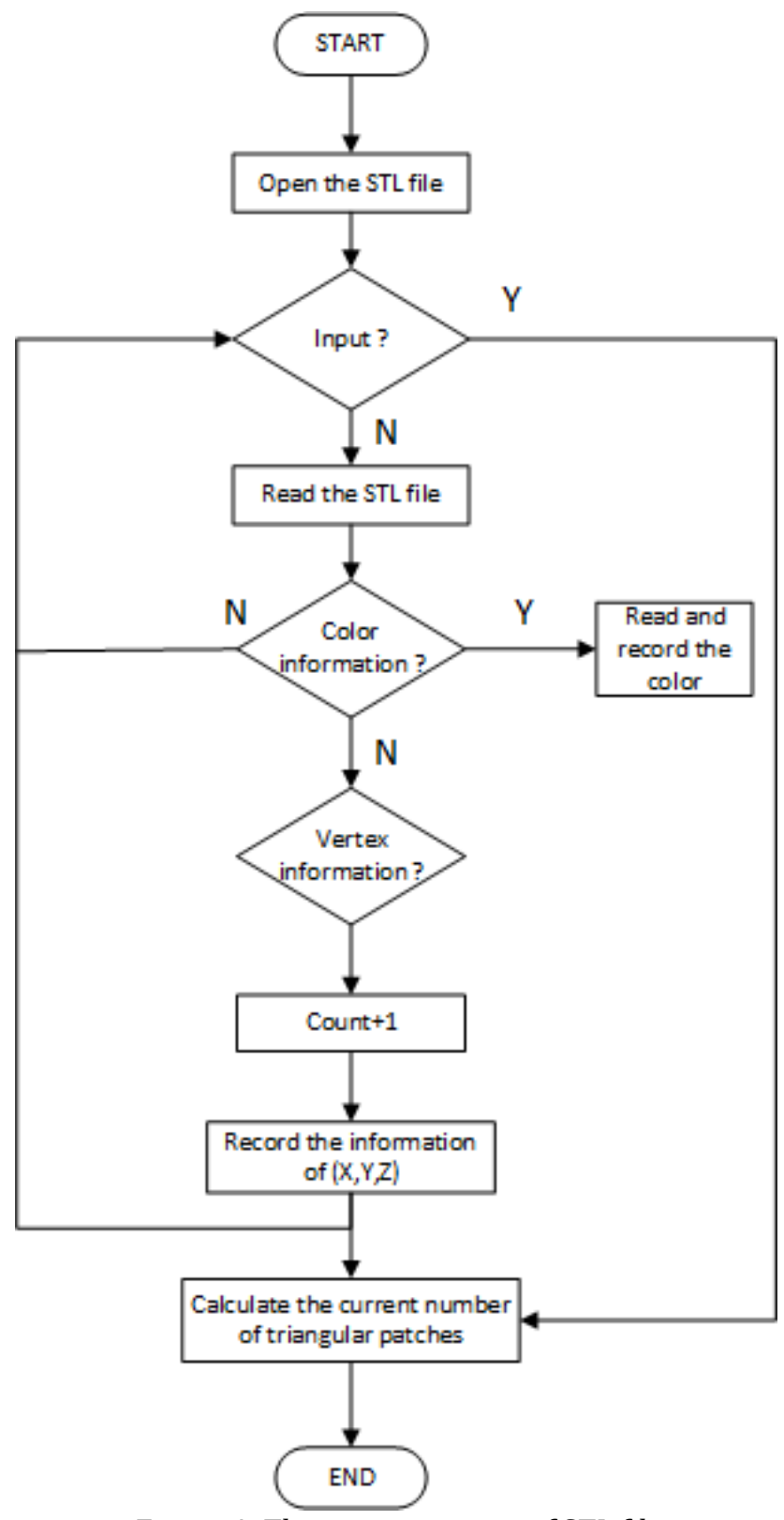

Figure 4: The import process of STL file

\subsection{Robot arm model display and animation demonstration}

After importing the generated STL files with specific ASCII format into MATLAB software, the model data stored in mat files is displayed through MATLAB 3D animation and display technology. In the visualization function of MATLAB, patch function is provided, and the calling format is:
handle=patch('Vertices' , propertyvalue ,

'Faces' , propertyvalue ......)

This function displays properties of the display object, such as 'Vertices' and 'Faces', and the 'Handle' is used to manipulate the displayed object. For the mechanical assembly, the displayed effect of the manipulator in MATLAB is shown in fig.5. 


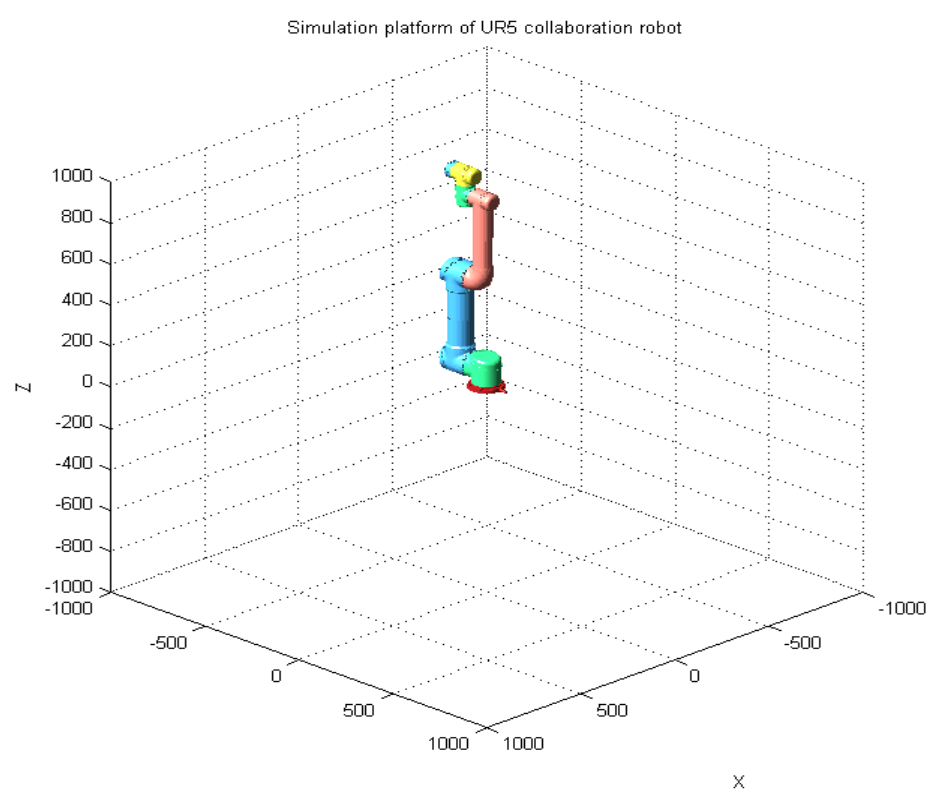

Figure 5: The display of the manipulator in MATLAB

As shown in fig.5, the posture after importing MATLAB is consistent with mechanical arm that in front, and the generation of STL file data is described in the new coordinate system, not like in the literature [15].

As a result, the rapid construction method of virtual simulation platform can effectively avoid the re-assembly process after import, and it only needs the coordinate system of each part.

In order to build a complete 3D virtual simulation platform for the mechanical arm, it is necessary to add movement characteristics, interactive interface and $3 \mathrm{D}$ animation.

According to the literature [3], the paper adopts the toolkit to cooperate with the parts, and writes the program in MATLAB software for 3D simulation platform.

Due to the less system resources and more interactive of the object, this paper adopts object mode to realize the animation demonstration of mechanical arm movement.

With the joint variables of the manipulator, the pose of the manipulator is calculated relatively. When DH parameters are applied to describe the pose relationship between the link coordinate system of the manipulator arm, the expression of transformation matrix between two adjacent links and the kinematics equation of the manipulator are as follows [16]:

$$
\begin{aligned}
{ }^{i-1} T_{i} & =\left[\begin{array}{cccc}
c \theta_{i} & -s \theta_{i} & 0 & \alpha_{i-1} \\
s \theta_{i} c \alpha_{i-1} & c \theta_{i} c \alpha_{i-1} & -s \alpha_{i-1} & -d_{i} s \alpha_{i-1} \\
s \theta_{i} s \alpha_{i-1} & c \theta_{i} s \alpha_{i-1} & c \alpha_{i-1} & d_{i} c \alpha_{i-1} \\
0 & 0 & 0 & 1
\end{array}\right] \\
{ }^{0} T_{6}(\theta) & =\prod_{i=1}^{6}\left({ }^{i-1} T_{i}\left(\theta_{i}\right)\right)={ }^{0} T_{1}{ }^{1} T_{2}{ }^{2} T_{3}{ }^{3} T_{4}{ }^{4} T_{5}{ }^{5} T_{6} \\
& =\left[\begin{array}{llll}
n & o & a & p \\
0 & 0 & 0 & 1
\end{array}\right]=\left[\begin{array}{cccc}
n_{x} & o_{x} & a_{x} & p_{x} \\
n_{y} & o_{y} & a_{y} & p_{y} \\
n_{z} & o_{z} & a_{z} & p_{z} \\
0 & 0 & 0 & 1
\end{array}\right]
\end{aligned}
$$

Where the parameter $c_{i}$ represents $\cos \left(\theta_{i}\right)$, $s_{i}$ represents $\sin \left(\theta_{i}\right), \theta_{i}$ is the joint variable, $p$ is the position vector of the end reference point relative to the base coordinate system, $[n, o, a]$ is the attitude matrix of the end reference point relative to the base coordinate system.

With the help of the robot toolbox, the motion characteristics of the robot arm are added to the simulation model, and a 3D virtual simulation platform of the robot arm integrating simulation model, interactive interface and kinematics characteristics can be built.

Taking the modular 6-dof manipulator as an example, the simulation model, interactive interface and some animated demonstration screenshots of motion are shown in fig.6 and fig.7, respectively. 


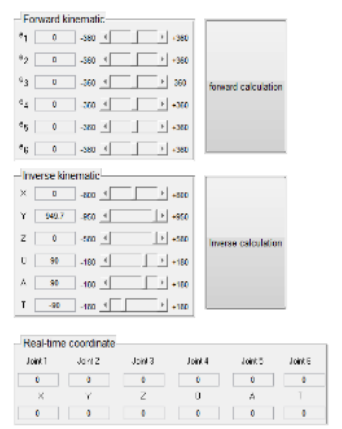

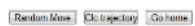

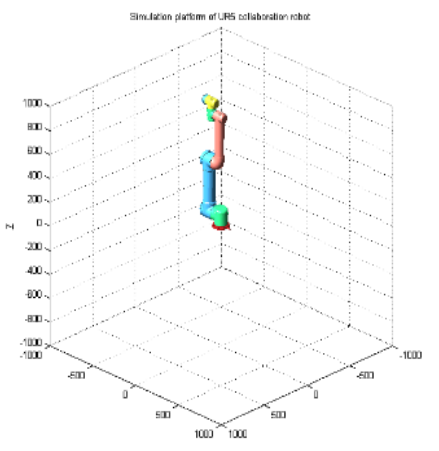

Figure 6: Simulation platform of the manipulator

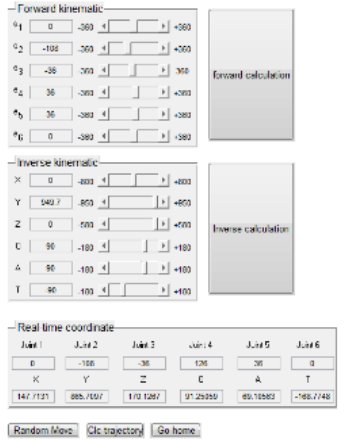

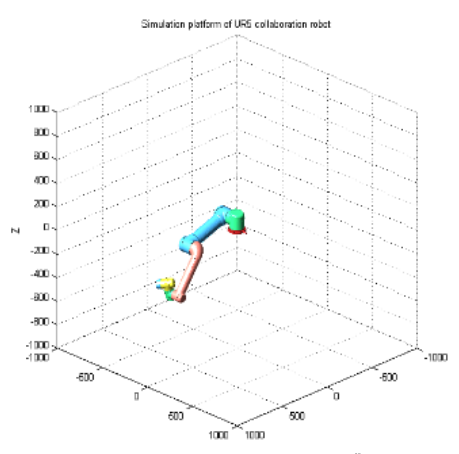
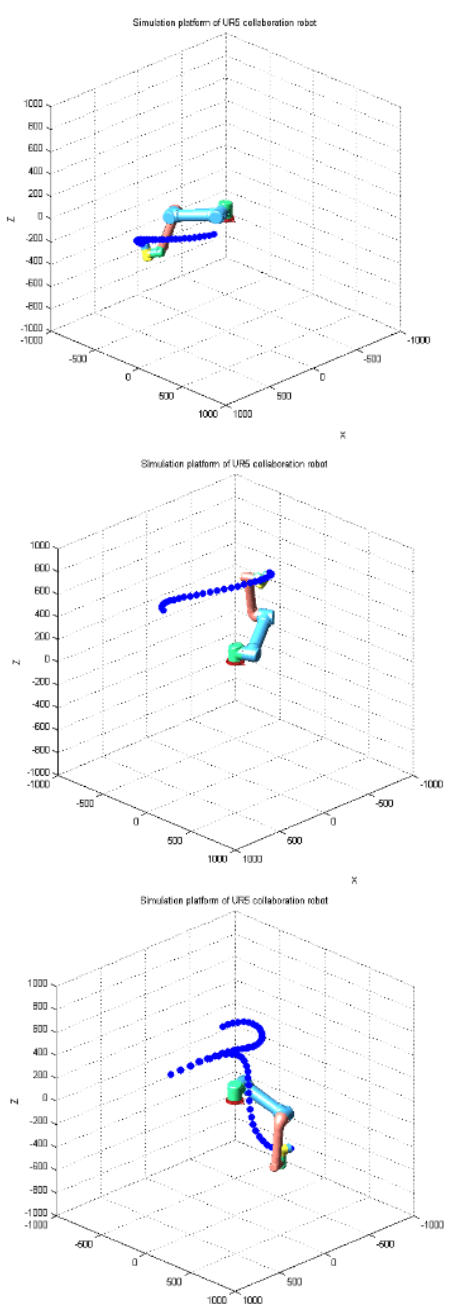
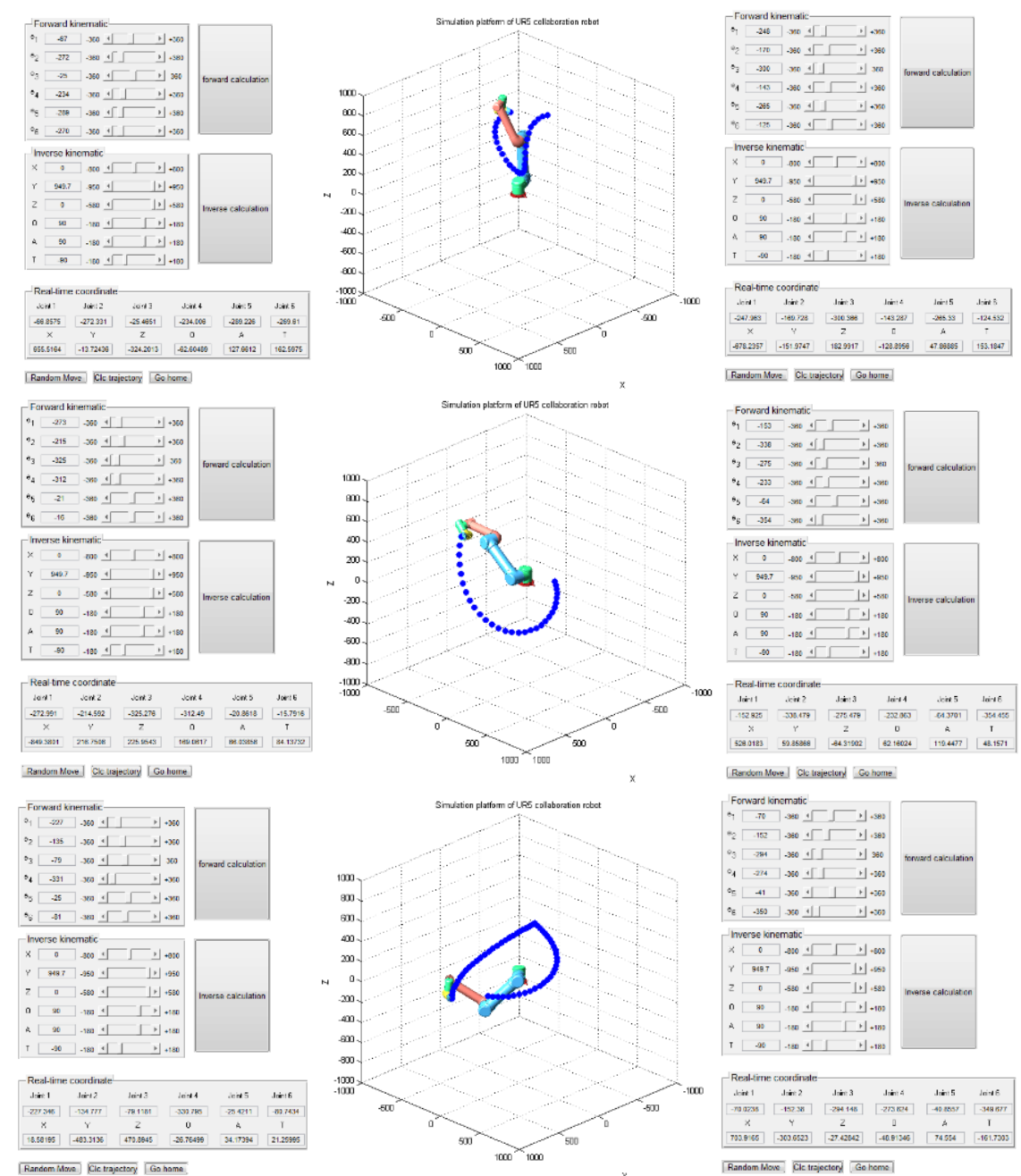

Figure 7: The animation of manipulators virtual simulation platform

As can be seen from fig. 6 and fig. 7, the poses of the model remain the same without chaos. The interactive interface can be shown in fig. 6 with the robot toolbox and the GUI interaction design. As the test reflects, it can quickly build a $3 \mathrm{D}$ virtual simulation platform for mechanical arm with interactive interface to meet the corresponding requirements. Fig. 7 shows a part of the movement demonstration of the 3D virtual simulation platform, and the movement of the mechanical arm is set as the random movement.

\section{The Avantages of this Method}

For the literature [15], the new method can avoid the problem with disorder distribution of each module and build the platform with higher efficiency. Compared with other robot simulation programs, the method has the following advantages:

(1) The method is based on the MATLAB software environment, fusing fully with Solid Works, robot toolbox, MATLAB GUI interaction design and MATLAB 3D graphics display technology to compile the $3 \mathrm{D}$ virtual simulation platform, and it reflects the 
high open degree, strong interactivity and well fidelity.

(2) This method is applied to the construction of 3D virtual simulation platform with various mechanical arm structures, and the standardized STL file that imported into MATLAB ensures that the development simulation program has an open type library of mechanical arm.

(3) The method with the robot kit can meet different design requirements. Not only avoiding the hard programming, but also omitting the basic operation process. In the future, it can contribute to a higher level of planning and the needs of teaching or other applications.

\section{Conclusion}

In this paper, a method that based on the literature [15] is proposed to build a mechanical arm open 3D virtual simulation platform. It combines Solid Works with MATLAB, especially the robot toolbox, GUI interaction design, MATLAB powerful numerical computation ability and 3D graphics display technology, to enhance functions of robot graphics and animation demonstration. In this paper, a 6-dof modular manipulator UR5 is as an example. The 3D virtual simulation platform is rapidly constructed with the method, and some motion demonstrations are given to verify the effectiveness to meet the requirements of teaching and scientific research.

This method not only overcomes the problem that the imported modules are disordered and dispersed in the world coordinate system in literature [15], but also has a high degree of openness, strong interaction and realistic display of simulation model. At the same time, the efficiency in building the platform and developing the simulation program is also well over other robot simulation programs.

\section{Acknowledgment}

This work was supported by Zaozhuang science and technology plan project (2019GX10) and Zaozhuang University doctoral research startup fund project (2018BS030).

\section{Reference}

[1] Kucuk S, Bingul Z. An off-line robot simulation toolbox [J].Computer Application in Engineering Education, 2010, 18(1):41-52.

[2] Kucuk S, Bingul Z. An offline simulation package for robotics education and industrial purposes [C]//11th IEEE International Conference on Methods and Models in Automation and Robotics. Miedzyzdroje, Poland: IEEE, 2005:653-658.
[3] Corke P I.MATLAB toolboxes: Robotics and vision for students and teachers [J].Robotics \& Automation Magazine, 2007, 14(4):16-17.

[4] Qassem M A, Abuhadrous I, Elaydi H. Modeling and simulation of 5DOF educational robot arm $[\mathrm{C}] / / 2$ nd IEEE International Conference on Advanced Computer Control. Piscataway, NJ, USA: IEEE, 2010:569-574.

[5] Toz M, Kucuk S. Dynamics simulation toolbox for industrial robot manipulator [J].Computer Application in Engineering Education, 2010, 18(2):319-330.

[6] Zhou F F, Fan X P, Ye Z. 3-D Virtual Robotics Model Generated by D-H Parameters [J].Journal of System Simulation,2006,18(4):947-950.

[7] Fu B, Hu F F, Chen L, et al. The Multi-robot Simulation Platform Research and Realize Based on OpenGL [J].Modular Machine Tool and Automatic Manufacturing Technique, 2016(1):20-23.

[8] Zhu T T, Cen Y W. Design of GUI Simulation Platform for Demolition Robot Based on Robotics Toolbox [J].Machine Tool and Hydraulics,2014,42(3):73-75.

[9] Yan Y J, Zhu Q D, Jiang C. OpenGL based Experimental platform for Simulation of Robot Arm Control System [J]. Computer Simulation, 2006, 23(8):252-257.

[10] Wang J M,Fu C L,Huang Y L,et al. Dynamics Simulation and Bionic Control Platform for Biped Robot with Matlab [J].Journal of system Simulation,2011,23(5):977-983.

[11] Ye Y,Su X Y,Cen Y W. Design of a visual simulation platform for a demolition robot based on Matlab GUI [J]. Journal of Hefei University of technology, 2016, 39(6):741-745.

[12] Sun Z Q, Yan J W, Fan X E et,al. Dynamic simulation of PUMA manipulator [J].Robot,1987,1(5):1-5.

[13] Xiong Z Y,Wang D X. Application of OpenGL in 3D animated simulation system of MOTOMAN arc-welding robot [J].Journal of Shanghai Jiaotong University,2008,42(S1):87-89.

[14] Chai T T, Ding Y L. The development of the robotics simulation and automation [J].Manufacturing Automation, 2011, 33(5):8789.

[15] Gan Y H, Dai X Z. An Efficient Method of Constructing Open 3D Simulation Environment for Articulated Robot [J].Robot, 2012, 34(5):628-633.

[16] Xiong Y L, Tang L X, Ding H,et al. Fundamentals of robot techniques [M].Wuhan: Huazhong university of science and technology press,2008. 